\title{
Research Paper \\ Providing Mindfulness-Based Educational Package for Evaluating Academic Procrastination
}

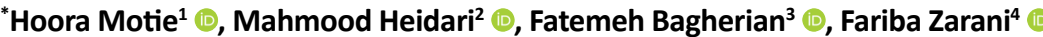

1. PhD. Candidate, Department of Psychology, Faculty of Education and Psychology, Shahid Beheshti University, Tehran, Iran.

2. PhD. in Educational Psychology, Associate Professor, Department of Applied Psychology, Faculty of Education and Psychology, Shahid Beheshti University, Tehran, Iran

3. PhD. in Psychology, Associate Professor, Department of Applied Psychology, Faculty of Education and Psychology, Shahid Beheshti University, Tehran, Iran.

4. PhD. in Psychology, Assistant Professor, Department of Clinical Psychology and Health, Faculty of Education and Psychology, Shahid Beheshti University, Tehran, Iran

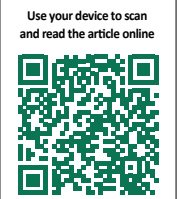

ciftation Motie H, Heidari M, Bagherian F, Zarani F. Providing Mindfulness-Based Educational Package for Evaluating Academic Procrastination. Iranian Journal of Psychiatry and Clinical Psychology. 2019; 25(1):26-41. http://dx.doi.org/10.32598/ ijpcp.25.1.25

doil http://dx.doi.org/10.32598/ijpcp.25.1.25

Received: 17 Oct 2018

Accepted: 04 Oct 2018

Available Online: 01 Apr 2019

Keywords:

Academic procrastination, Mindfulness,

Self-efficacy, Test anxiety, Self-esteem, Perfectionism

\section{A B S T R A C T}

Objectives Academic procrastination is an unreasonable delay in the submission of academic assignments, which is very common. This research was designed to develop and study the effect of a mindfulness-based educational package on reducing academic procrastination.

Methods The educational package based on mindfulness for reducing academic procrastination was designed by using mindfulness-based techniques and the relevant literature of academic procrastination considering the effective constructs, including self-efficacy, test anxiety, self-esteem, and perfectionism. In a quasi-experimental study, 36 subjects were selected. In order to study the effect of the educational package on students, the experimental and control groups were put into pre-test, post-test, and followup categories.

Results Mixed ANOVA revealed the effectiveness of the provided educational package on improving mindfulness $(F=20.87 ; P<0.001)$ and reducing academic procrastination $(F=44.67 ; P<0.001)$ whose effect was significant.

Conclusion Based on the results, self-efficacy, test anxiety, self-esteem, and perfectionism of the participants in the post-test and follow-up were significantly different from the pre-test. The findings of this study are consistent with the findings of previous studies. These results are applicable to clinical and educational practitioners.

\section{Extended Abstract}

\section{Introduction}

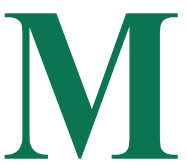

any students believe that their low scores (from the expected level) are due to their inability to complete academic assignments within a given time frame. Procrastination leads to increased stress, disorder, and failure. The procrastination behavior is obvious among students, which can be observed significantly among those students who seek advisers.

Among new approaches, acceptance and commitment therapy and mindfulness-based therapy have drawn much attention. Based on the metacognitive awareness theory, difficult assignments can activate biases and defensive thoughts which encourage disappointment, self-criticism, and impul-

* Corresponding Author:

Hoora Motie, PhD. Candidate

Address: Department of Psychology, Faculty of Education and Psychology, Shahid Beheshti University, Tehran, Iran.

Tel: +98 (21) 22041635

E-mail: hu.motie@gmail.com 
sive decisions that end in avoiding the assignment. Instead, the mindfulness facilitates the confirmation and acceptance of these challenging thoughts and prevents mind confusion. Since procrastinators seek to achieve instant calm after experiencing a stressful assignment, low mindfulness may be regarded as another contributing factor to avoidance, which is usually related to procrastination. However, a large body of research indicates that procrastinators are highly resistant to change and may only respond positively to interventions based on the readiness for change. It seems that among the third wave interventions, the mindfulness can be effective in reducing the procrastination.

As procrastinators are resistant to change, the present study aimed to develop an educational package by investigating the structures affecting the academic procrastination, such as test anxiety, self-esteem, self-efficacy, perfectionism, and using mindfulness-based techniques. To the best of our knowledge, no mindfulness-based interventional plan has been ever performed to reduce academic procrastination. Thus, it seems that the present study is the first project in this field.

\section{Method}

Study subjects

In the present study, 36 students were willing to participate in the research. They were randomly selected and assigned to the experimental and control group ( $n=16$ for each) after meeting the related inclusion and exclusion criteria. The inclusion criteria were being between 18 and 35 years old and studying in undergraduate or masters' degrees. The exclusion criterion was missing the training program even for one session. The subjects in both groups responded to the questionnaires before the start of the program, immediately after the end of the program, and one month after the end of the program. The training package was implemented during 8 sessions in a one-month course on the subjects of the experimental group, and the instructor committed to conducting this course for the control group in the next few months.

\section{Developing the educational package}

The mindfulness-educational package was studied and selected to reduce academic procrastination and extract common elements and principles of educational and therapeutic methods of mindfulness, research backgrounds, and researcher's findings in the study of predictor factors of academic procrastination and existing mindfulness-based guidelines which were designed by experts in Iran and outside of Iran. Then, the objectives, content of the sessions and assignments, and their exercises were evaluated.

By combining and matching exercises and assignments with the factors related to academic procrastination, a guide was formulated entitled "Mindfulness Training Guide", aiming at increasing mindfulness and reducing the academic procrastination. After designing the package, its copies were provided to five clinical psychologists with a $\mathrm{PhD}$. degree and asked them to answer two questions related to the purpose and timing of the sessions. Then they provided their comments and suggestions concerning the purpose and duration of each session based on a Likert-type scale. Finally, the modifications intended by evaluators were conducted.

\section{Results}

In this section, first, the effect of the devised package on mindfulness was studied. Then, the effect of the package on the self-efficacy, self-esteem, perfectionism and test anxiety variables was assessed. Finally, the effect of the package was measured on the academic procrastination.

\section{Mindfulness}

In the present study, the Independent t-test method was used to determine the significance of the total mindfulness score in the pre-test. The difference in mindfulness score with an average of 108 in the experimental group and 109.8 in the control group was not significant in the pretest $(\mathrm{P}=0.59, \mathrm{t}=-0.54)$. The effectiveness of the educational package on the mindfulness score was studied by mixed Analysis of Variance (ANOVA). The results of the Greenhouse-Geysers test indicated that the main effect of the

Table 1. Mixed Anova results for procrastination scores

\begin{tabular}{ccccccc}
\hline Source & Sum of Squares & df & Mean Square & F & Sig. & Partial Effect \\
\hline Time & 201.19 & 1.65 & 121.45 & 54.15 & $<0.001$ & 0.615 \\
\hline TimexGroup & 166.01 & 1.65 & 100.21 & 44.67 & $<0.001$ & 0.659 \\
\hline Error & 104.04 & 46.38 & 2.24 & & & \\
\hline
\end{tabular}


group, the condition of the test (pre-test, post-test, followup) and the group and condition interaction were significant with the effect size of $0.427(\mathrm{~F}=20.87, \mathrm{P}<0.001)$.

Self-efficacy, Self-esteem, Test anxiety, and perfectionism

The effectiveness of the educational package on each of the self-efficacy, self-esteem, test anxiety, and perfectionism variables were analyzed separately by using multivariate ANOVA. In order to evaluate the effectiveness of the experimental test, the difference between the post-test and the follow-up from the pre-test was calculated. The difference between the experimental and control groups was significant regarding the variables of test anxiety, self-efficacy, perfectionism, and self-esteem $(\mathrm{P}<0.001)$, i.e. the average test anxiety and perfectionism in the experimental group reduced by 2.75 and 2.4 points after the implementation of the educational package, while the self-efficacy and selfesteem increased by 1.87 points.

\section{Academic procrastination}

In order to analyze the significance of academic procrastination in the pre-test, the Independent t-test method was used. The effectiveness of the educational package on general procrastination was investigated by mixed ANOVA. The results of the Greenhouse-Geysers test indicated that the main effect of the group, the condition of the test (pre-test, post-test, follow-up) and the group and condition interaction were significant with the effect size of 0.659 $(\mathrm{F}=44.67, \mathrm{P}<0.001)$ (Table 1).

\section{Discussion}

Based on the results, the mindfulness-based educational package was significantly effective in increasing self-esteem, self-efficacy, and reducing test anxiety, perfectionism and, consequently, decreasing academic procrastination. These findings support previous research results. Since exercises based on mindfulness techniques continue after the end of the period and the individual was supposed to perform these exercises outside the group, as expected, it was observed that after one-month follow-up, changes were significantly consistent, indicating the consistency of the therapeutic effect.

Generally, the theoretical and research fundamentals related to the effectiveness of mindfulness-based techniques on reducing academic procrastination and the results of applying educational package provided in this study have verified the effect of this package. It is suggested that future research compare the mindfulness-based educational pack- age with traditional cognitive-behavioral methods in reducing academic procrastination.

\section{Ethical Considerations}

\section{Compliance with ethical guidelines}

All ethical principles were considered in this article. The participants were informed about the purpose of the research and its implementation stages; they were also assured about the confidentiality of their information; Moreover, They were allowed to leave the study whenever they wish, and if desired, the results of the research would be available to them.

\section{Funding}

This research was extracted from the $\mathrm{PhD}$. thesis of the first author, in Department of Psychology, Faculty of Educational Science and Psychology, Shahid Beheshti University, Tehran, Iran.

\section{Authors contributions}

Conceptualization: All authors; Methodology: Mahmood Heidari, Hoora Motie; Analysis: All authors; Research: All authors; Sources: Hoora Motie; Writing draft: Hoora Motie; Editing and finalizing: Hoora Motie; and Project management: All authors.

\section{Conflict of interest}

The authors declare no conflict of interest. 


\title{
ساخت و بررسى اثر بسته أموزشى مبتنى بر ذهن آتاهى كاهش اهمال كارى تحصيلى
}

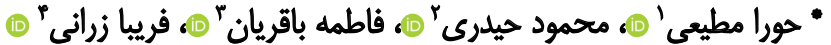

\begin{abstract}
1 - دانشجوى دكترا، كروه روانشئاسى، دانشكده علوم تربيتى و روانشناسى، دانشكاه شهيد بهشتى، تهران، ايران.

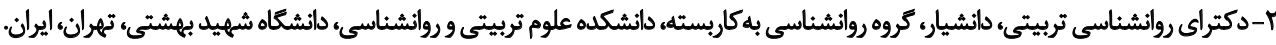

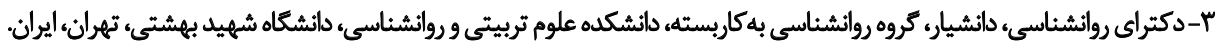

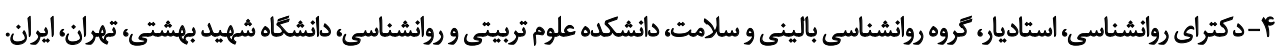

\begin{abstract}
حكند

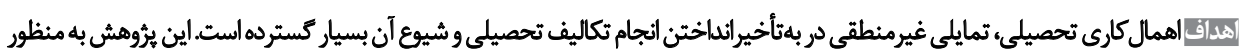

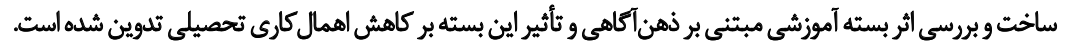

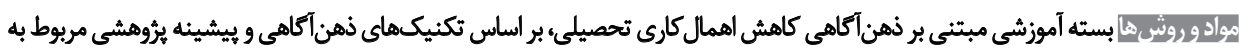

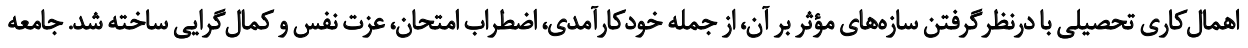

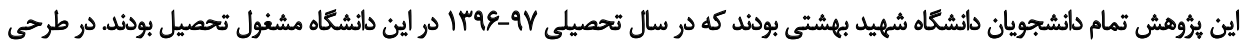

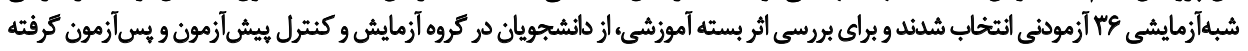

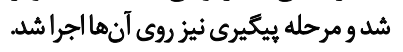

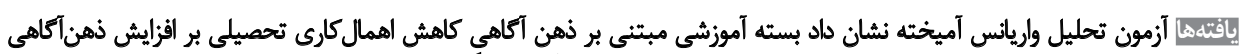

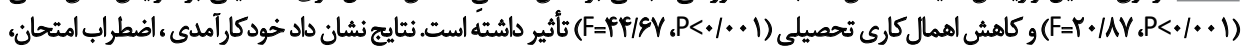

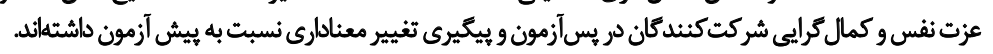

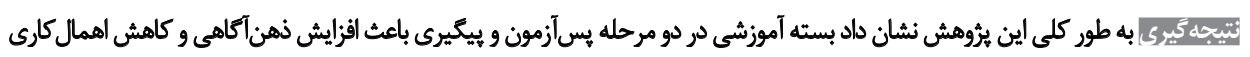

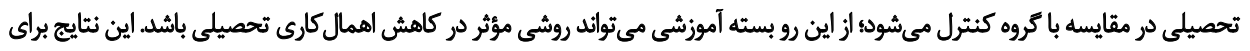

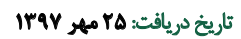

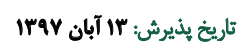

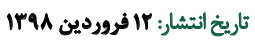

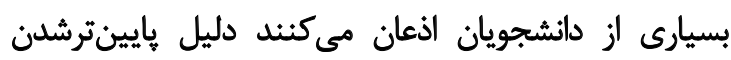

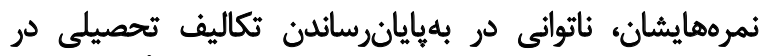

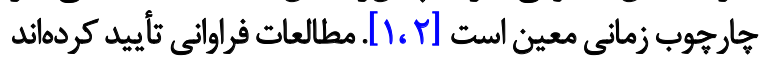
از بيامدهاى اهمال كارى استرس ذذهنى و فيزيكى، مشكلات

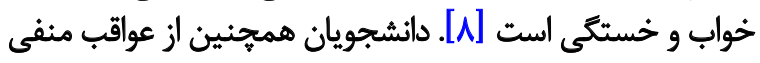

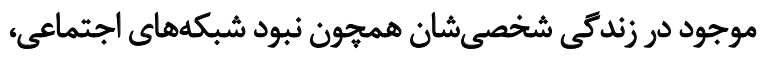

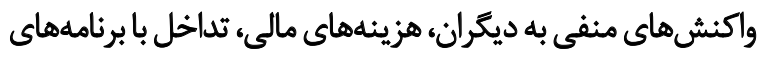

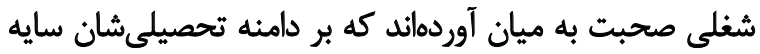

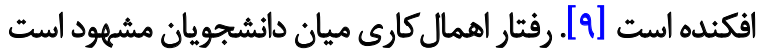

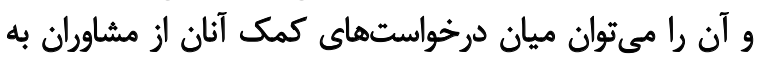

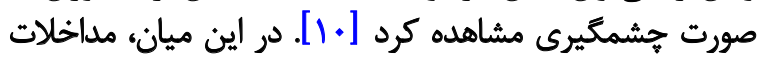

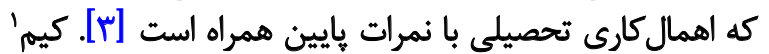

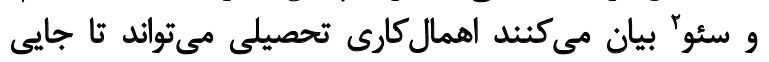

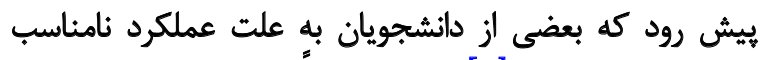

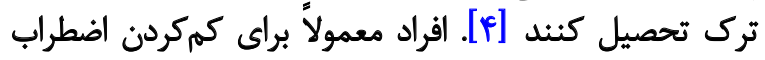

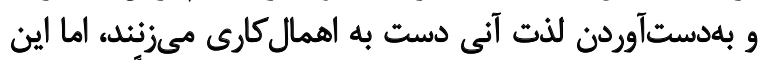

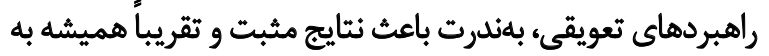

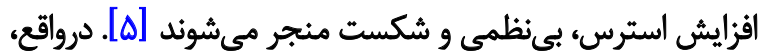

\section{Kim \\ 2. Seo}


به اهمال كارى است. ذهن آكاهى، استرس را كاهش و بهزيستى كئى

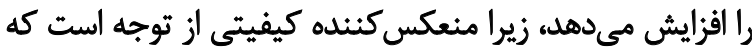

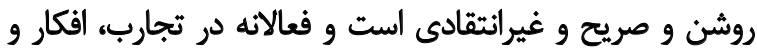

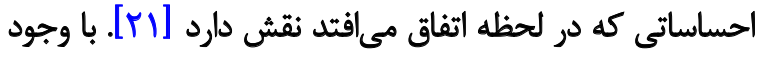

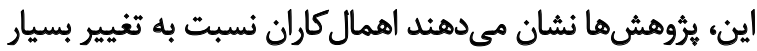

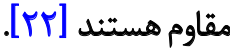

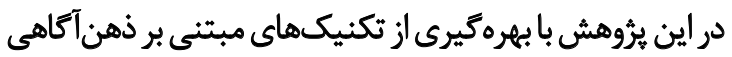

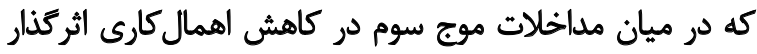

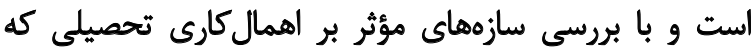

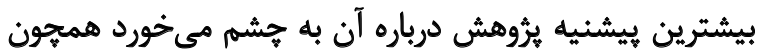

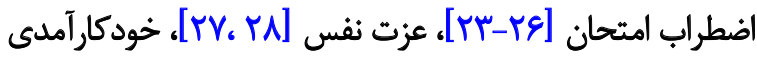

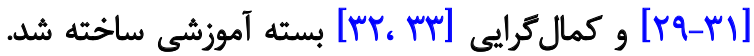

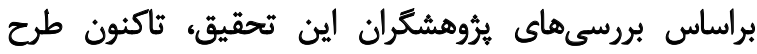

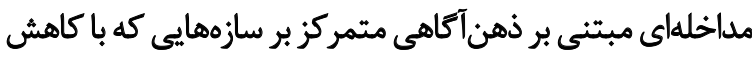

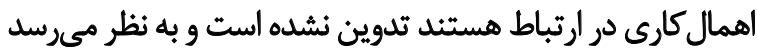
اين يروهش اولين طرح موجود در اين حوزه باشد.

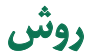

اين ثروهش در دستهبندى بر مبناى هدف، از نوع تحقيقات

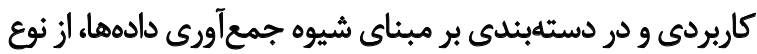

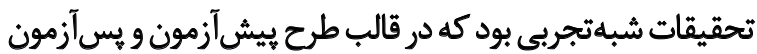

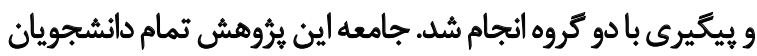

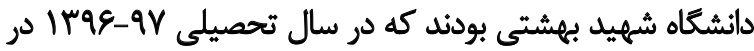

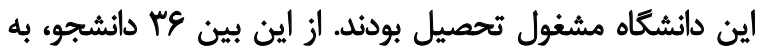

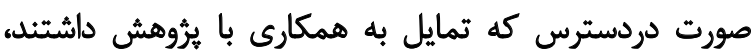

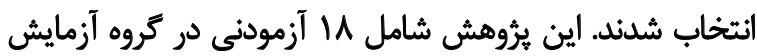

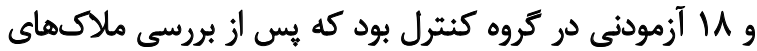

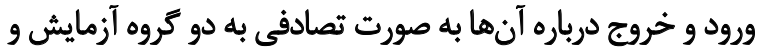
كنترل تقسيم شدند.

ملاكهاى ورود شركت كنندهها عبارت بودند ازي دامنه سنى بين

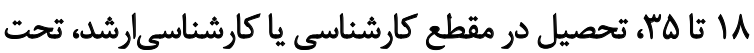

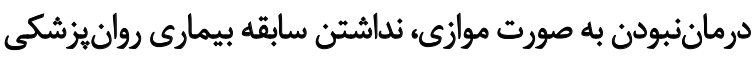

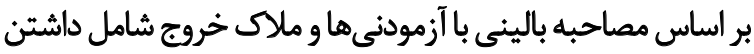

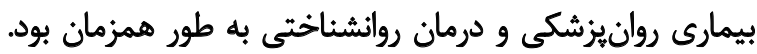

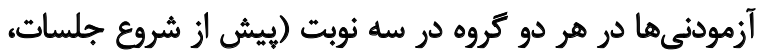

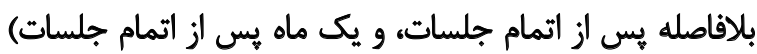

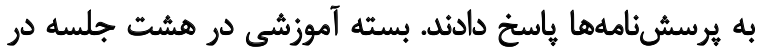

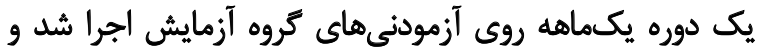

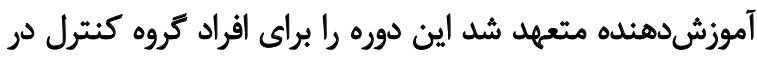
جند ماه آينده بركزار كند (جدول شماره آندار ).

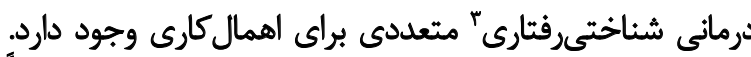
مداخلات درمانى شناختى فيتئي

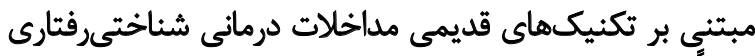

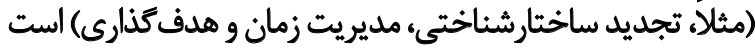

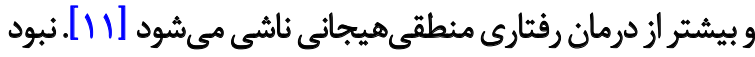

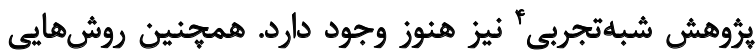

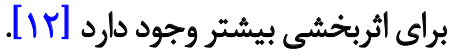

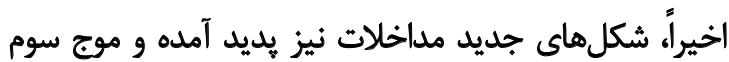

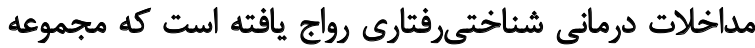

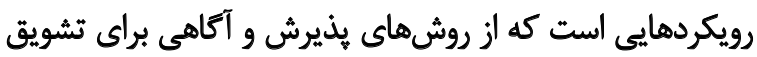

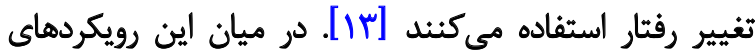

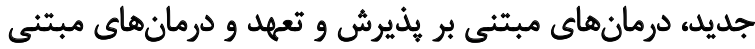

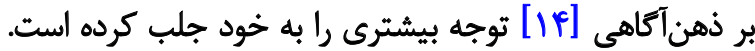

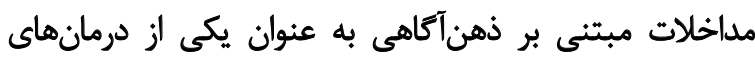

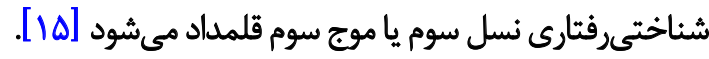
كابات زينه بيان مى دارد كه در ذهنآكاهى از طريق مشاهده

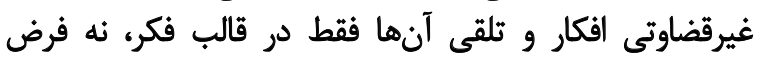

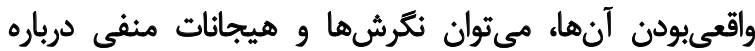

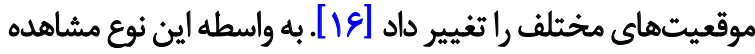

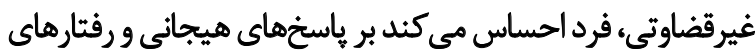

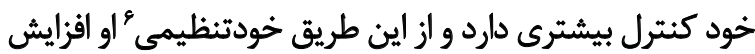

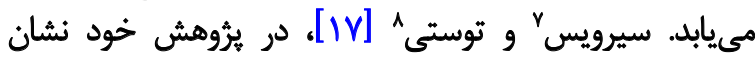

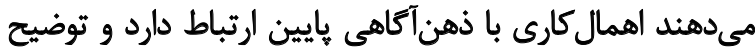

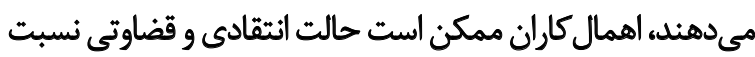

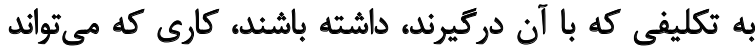

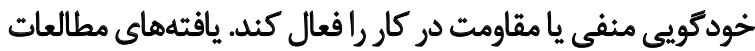

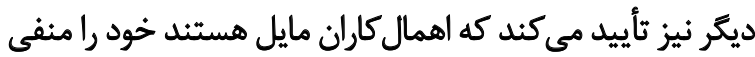
ارزيابى كنيند [11]

بر اساس نظريه آكاهى فراشناختى [19 [ ] تكاليف دشوار مى تواند

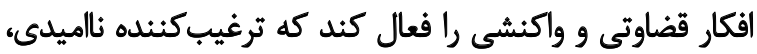

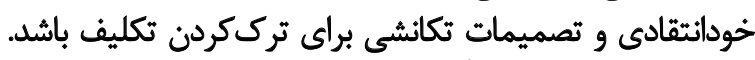

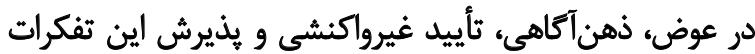

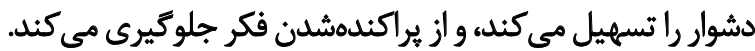

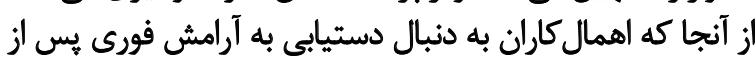

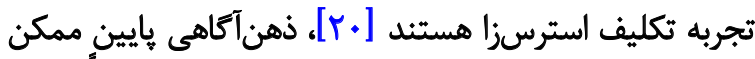

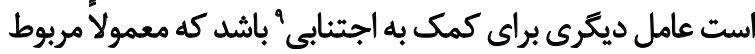

3. Cognitive Behavioral Therapy (CBT)

4. Quasi-experimental

5. Kabat-Zinn

6. Sense of self-efficacy

7. Sirois

8. Tosti

9. Avoidance 


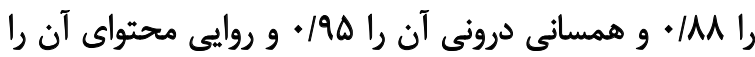

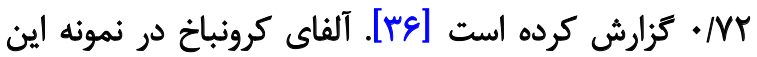

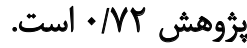

\section{مقياس هندبُعدى كمال كرايى"}

مقياس جندبعدى كمال كرايى يرسشنامهاى است كه در سال

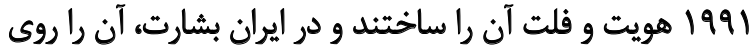

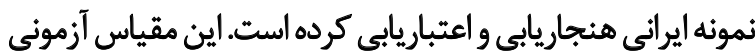

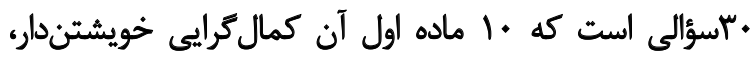

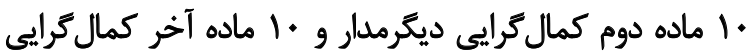

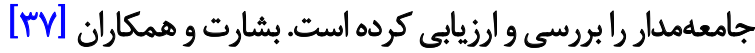

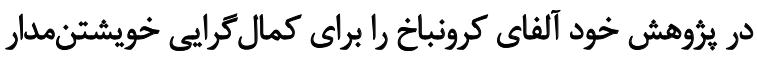

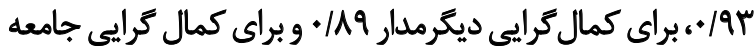

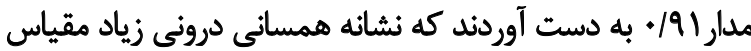

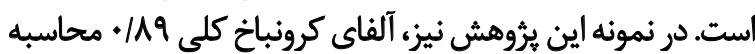

$$
\text { شده است. }
$$

\section{يرسش نامه خودكار آمدى شرر و همكاران"}

اين مقياس كه براى خودكارآمدى در نظر كرفته شده است

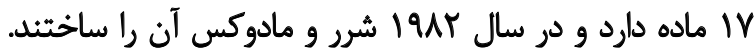

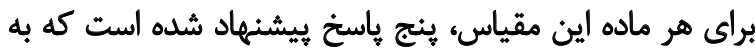

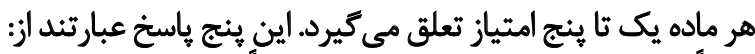

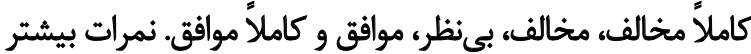

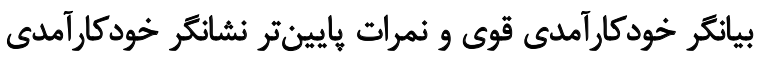

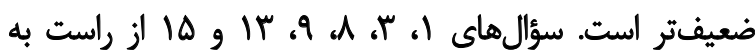

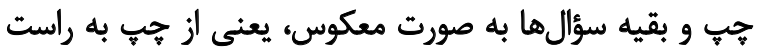

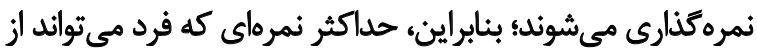

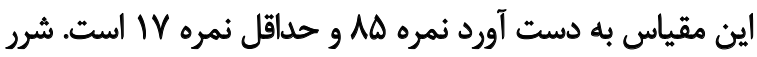

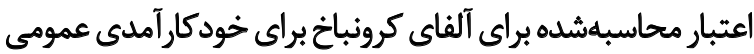

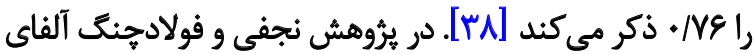

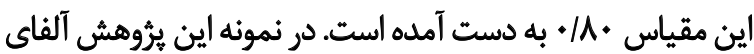

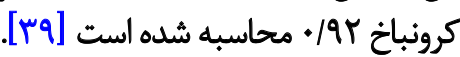

\section{مقياس عزت نفس روزنبرى"}

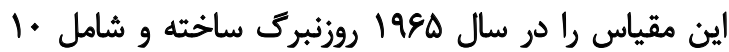

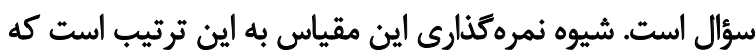

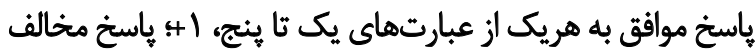

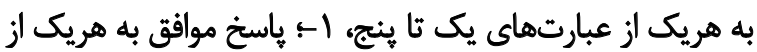

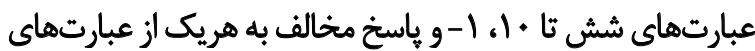

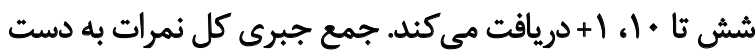

12. Multidimensional Perfectionism Scale (MPS)

13. Sherrer Self-Efficacy Scale

14. Rosenberg's Self-Esteem Scale (RSEC)

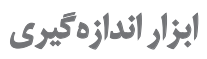

\section{يرسش نامه اهمال كارى تحصيلى سولومون و راثبلوم"}

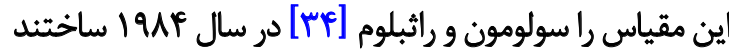

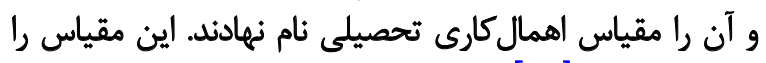

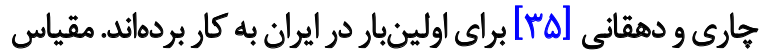

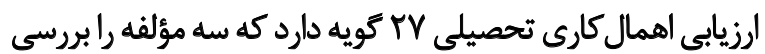

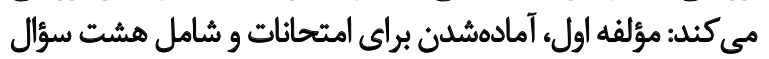

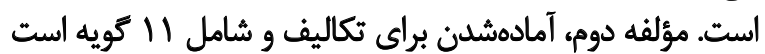

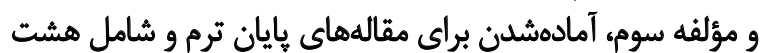

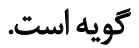

نحوه ياسخدهى به كويهها به اين صورت است كه باسخدهندكان

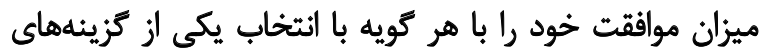

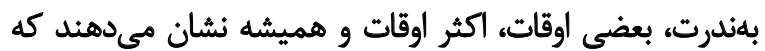

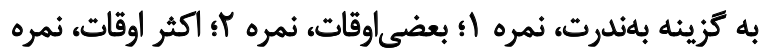

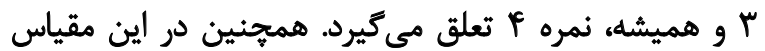

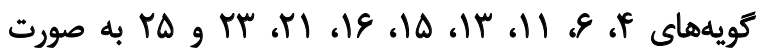

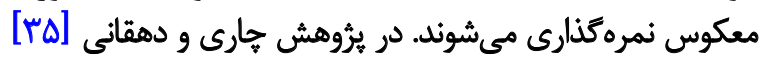

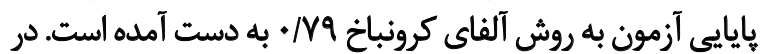

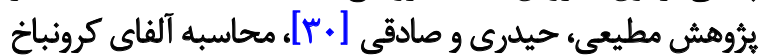

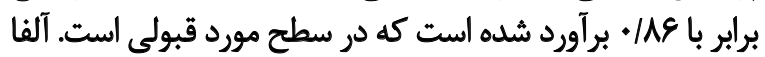

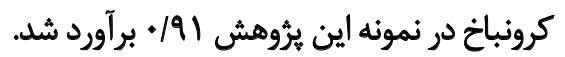

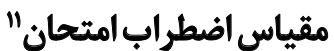

از جمله ابزارهايى كه براى ارزيابى و تشخيص اضطراب امتحان

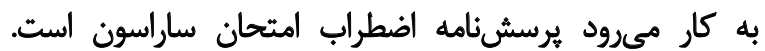

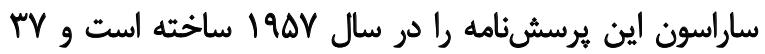

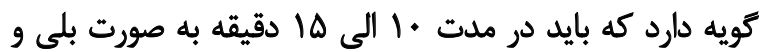

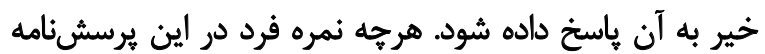

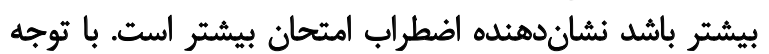

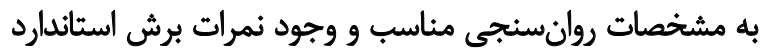

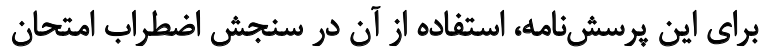

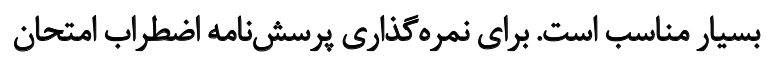

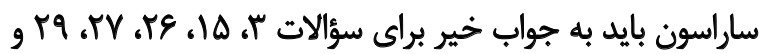

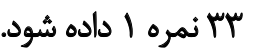

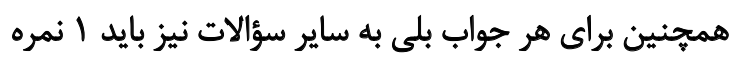

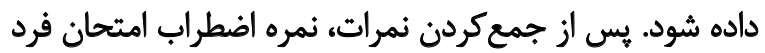

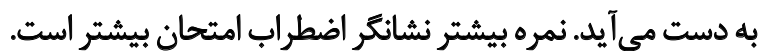

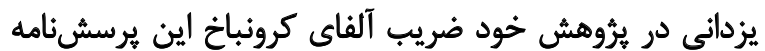

10. Solomon and Rothblum Procrastination Assessment Scale for Students

11. Test Anxiety Scale 
متوسط و در همه موارد معنى دار بود و در طيفى بين هار /. تا

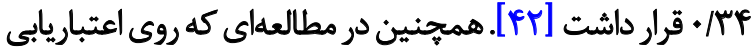

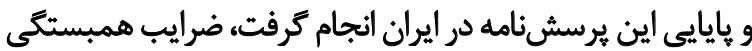

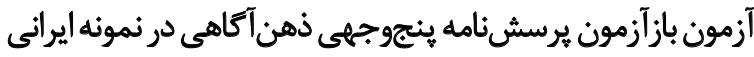

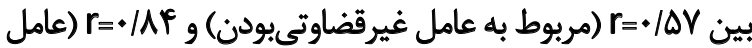

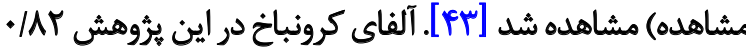
محاسبه شده است.

روش ساخت بسته أموزشى

بسته آموزشى مبتنى بر ذهن آكاهى به منظور كاهش اهمال كارى

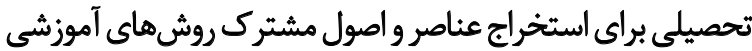

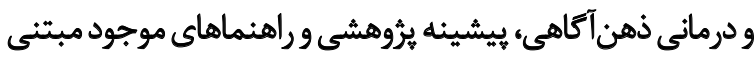

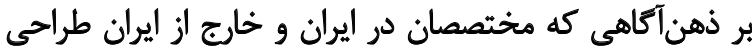

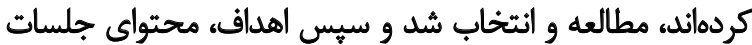

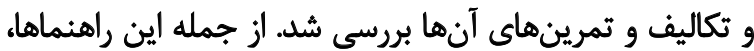

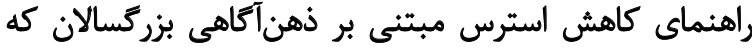

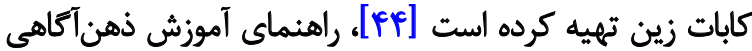

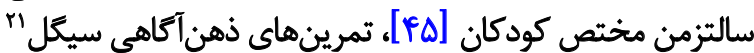

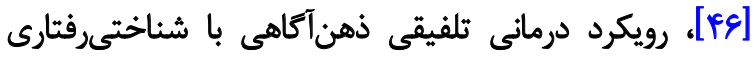

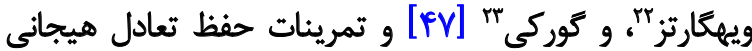

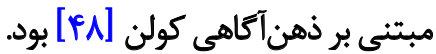

يكى از عناصر مشترك و اصلى در تمامى اين راهنماها، بودن در

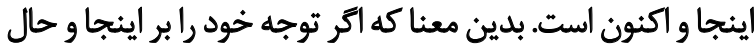

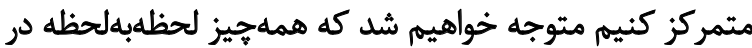

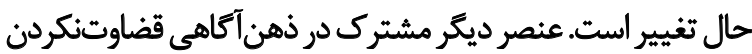

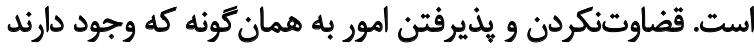

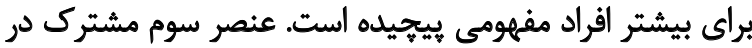

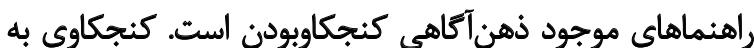

21. Siegel

22. Wiegartz

23. Gyoerkoe

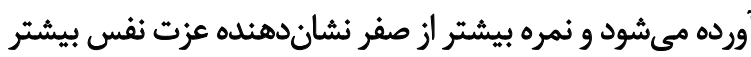

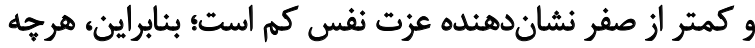

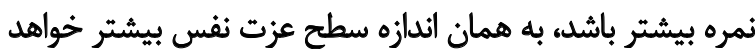

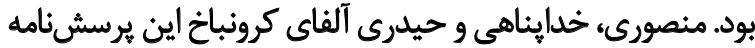

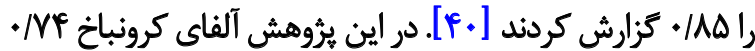

$$
\text { برآورد شده است. }
$$

\section{يرسشنامه ينجوجهى ذهن آتاهى ها}

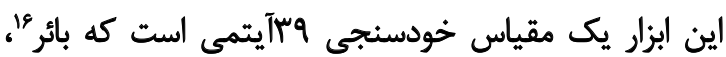

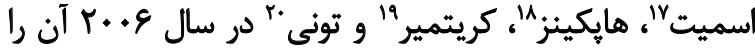

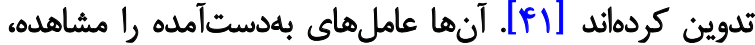

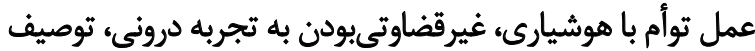

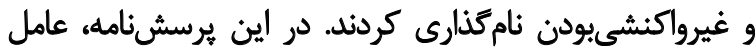

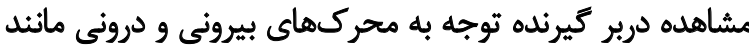

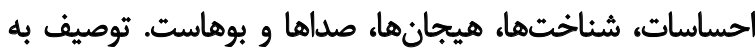

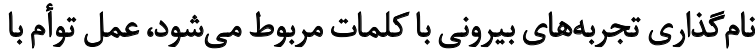

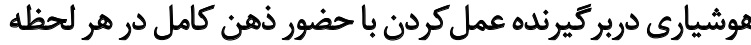

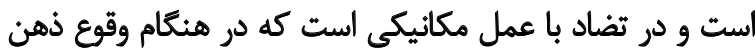

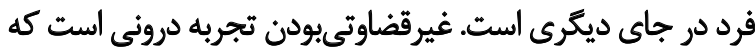

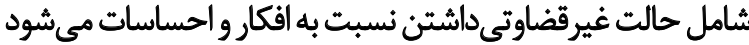
و غيرواكنشى بودن به تجربه درونى آمد و رفت به افكار و واحت احساسات درونى كفته مى شود، بى آنكه فرد در آنها كير كندي

بر اساس نتايج، همسانى درونى عاملها مناسب بود و ضريب

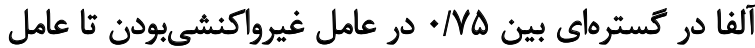

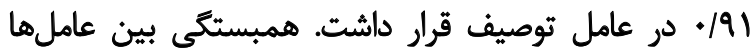

15. Five Facet Mindfulness Questionnaire (FFMQ)

16. Baer

17. Smith

18. Hopkins

19. Krietemeyer

20. Toney

جدول ا. خلاصه بسته آموزش مبتنى بر ذُهن آكاهي به منظور كاهش اهمال كارى تحصيلى

$$
\text { هحتواي جلسات (تمرينهاي جلسه) }
$$$$
\text { هدف جلسه }
$$$$
\text { جلسه }
$$

معرفي شركت كنثدكان به يكديكر ذهن أكاهي: معرفى كتى تمرين واكتش در برابر تغييرات تمرين توقف در برابر فكر برين تمرين فهرست افكار منفى دورئ

تمرين كوشسيارى

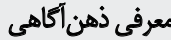
تمرين خوردن كشمش دمن

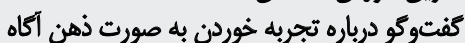
ذهن أكاهي و ادراك زمان

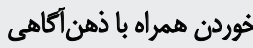
تقويت ذهن أكاهى دوم: خوردن و تنفس خورن 


\begin{tabular}{|c|c|c|}
\hline محتواي جلسات (تمرينهاي جلسه) & هدف جلسيه & جلسه \\
\hline 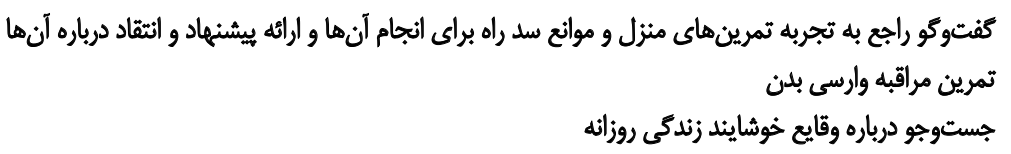 & 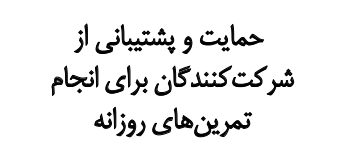 & سوم: شروعى \\
\hline 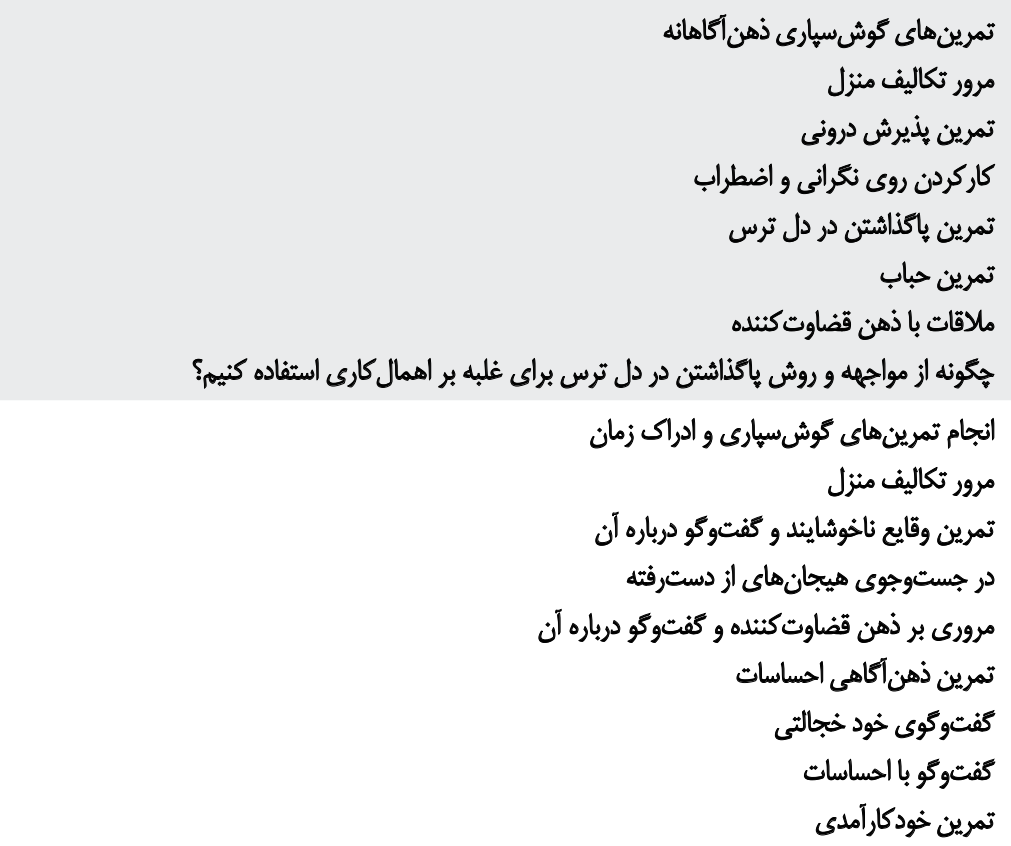 & 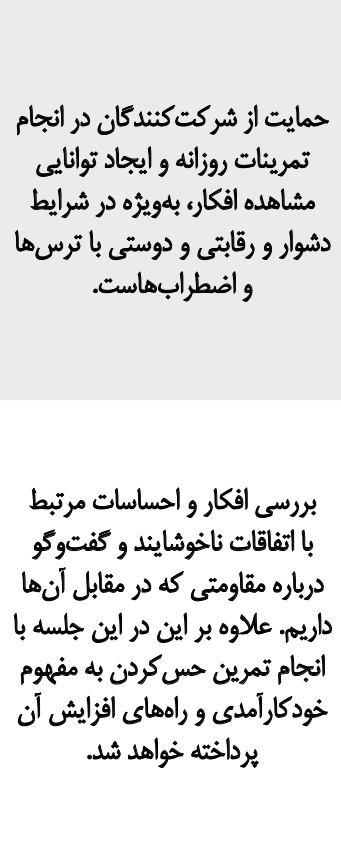 & 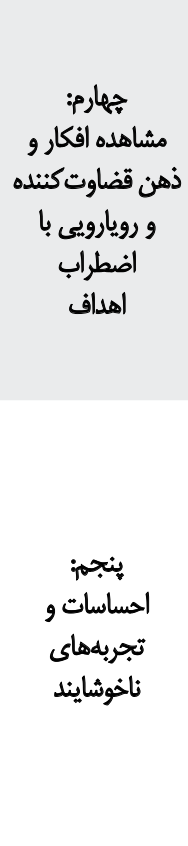 \\
\hline 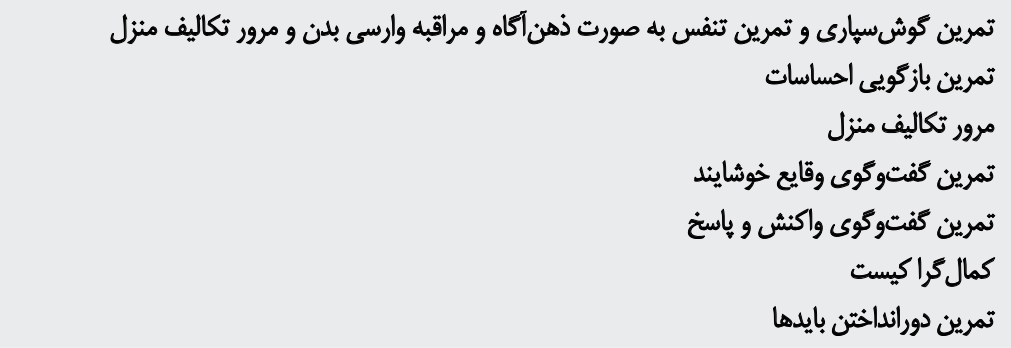 & 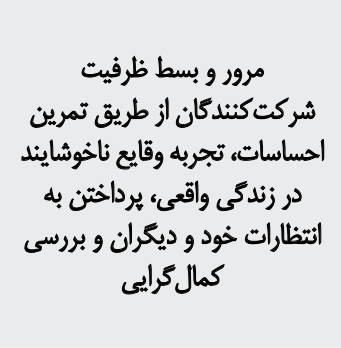 & عكسم: يَاستخ و \\
\hline 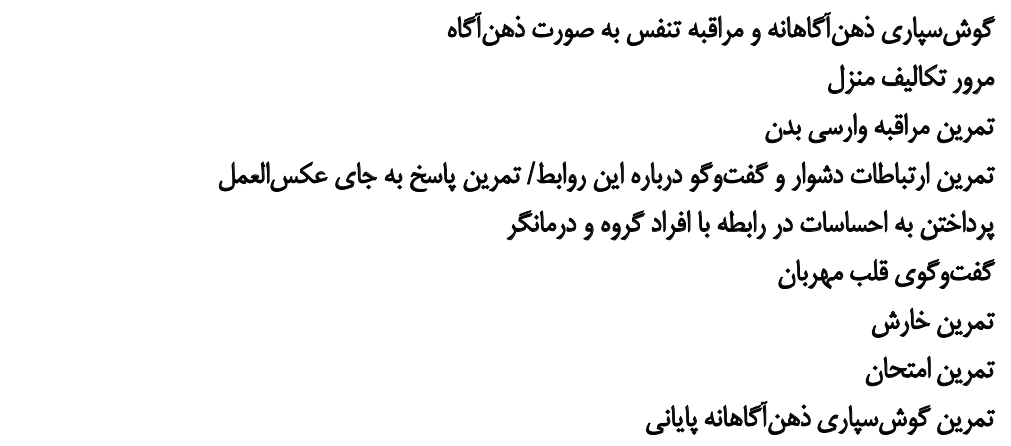 & 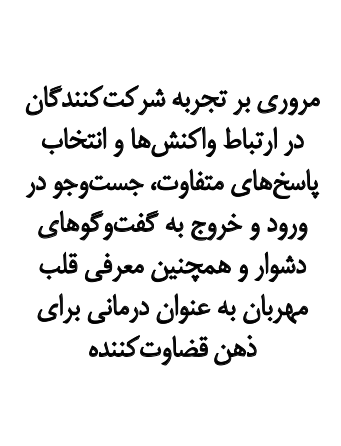 & هفته: واكنش و \\
\hline 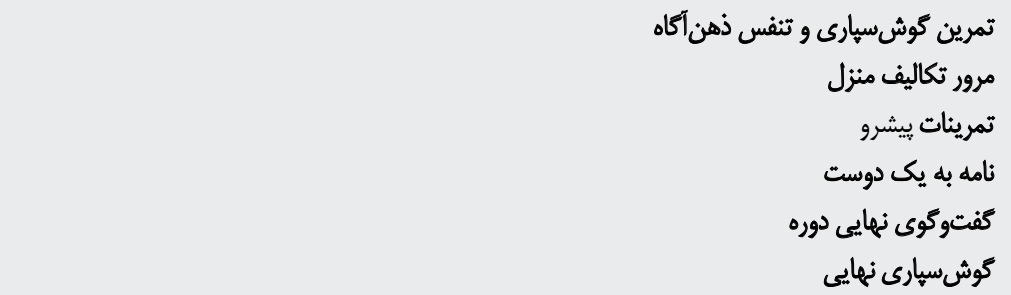 & 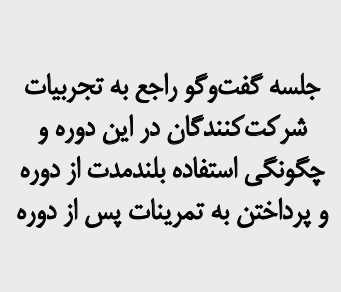 & هشتم: اتمام دوره \\
\hline
\end{tabular}


به آن بنويسند. نتايج نشان داد ضريب همبستكى درونى نظرات

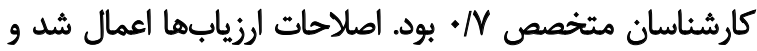
تغييراتى كه نياز بود صورت كرفت

ياقتثهها

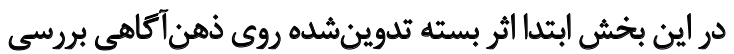

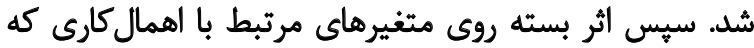

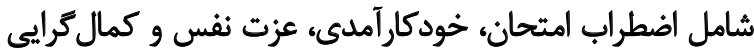

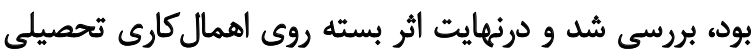

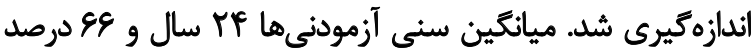

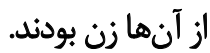

$$
\text { ذهن أكَاهـ }
$$

ابتدا براى بررسى معنادارى نمره ذهن آكاهى كل در بيش آزآزمون

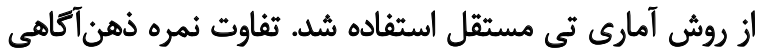

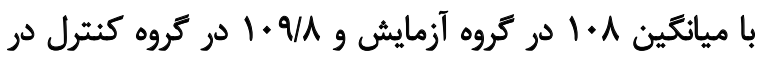

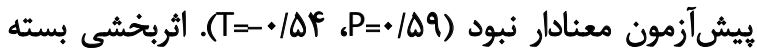

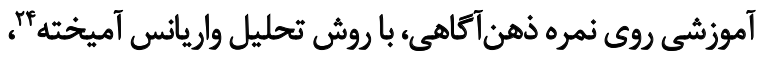

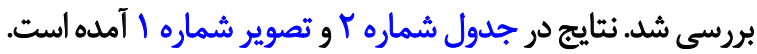

24. Mixed ANOVA

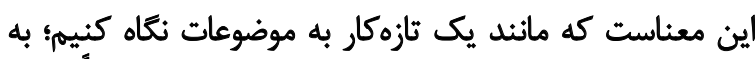

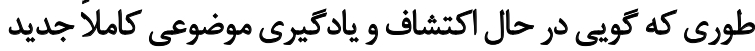

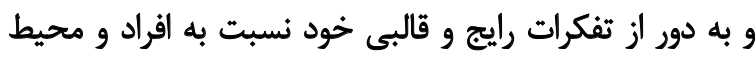

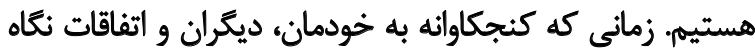

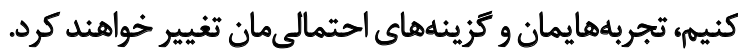

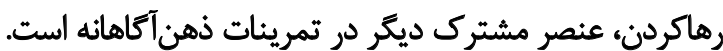

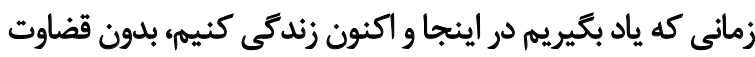

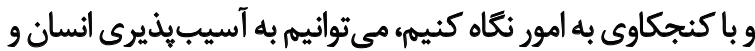

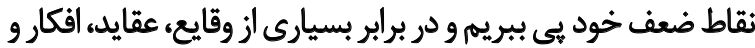

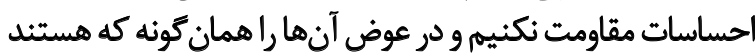

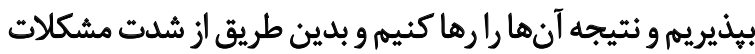
كم يا مسائل را به طور صحيح حل كنيه.

با توجه به موارد مذكور، تركيب و تطبيق تمرينها و تكاليف با

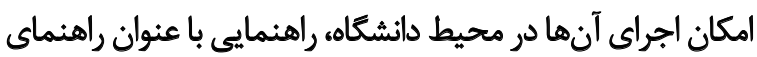

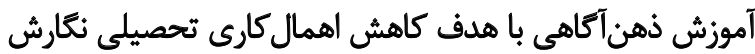

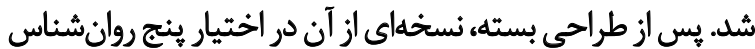

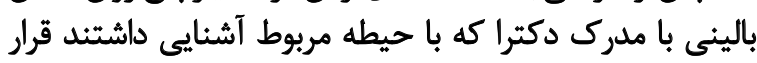

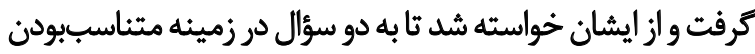

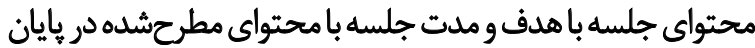

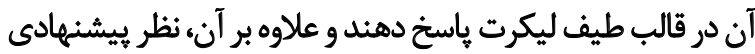

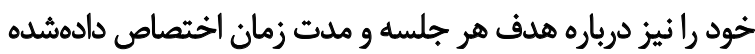

جدول r. نتايج تحليل واريانس آميخته براى نمره كلى ذهن آكاهى

\begin{tabular}{|c|c|c|c|c|c|c|}
\hline الدازه اثر & سطح معنادارى & $\mathbf{F}$ & ميانكين مجذورات & درجه آزادى & مجموع مجذورات & منيع واريانس \\
\hline.$/ 29$ & $<+1 \cdot+1$ & WA & $n \in / 4 \lambda$ & $1 / 4$ & $1 .+/ q f$ & موقعيت \\
\hline \multirow[t]{2}{*}{.$/ F T Y$} & $<+1++1$ & $r \cdot / A V$ & Af & $1 / 4$ & WWHE & موقعيت Xكروه \\
\hline & & & $r / . r$ & PVIFD & IQNVE & خطا \\
\hline
\end{tabular}

جدول r. جدول تحليل واريانس جندمتغيرى تغيير اضطراب امتحان، خودكارآمدى، كمال كرايى، عزت نفس در يسآزمون

\begin{tabular}{|c|c|c|c|c|c|c|c|}
\hline سطح معنادارى & اندازه اثر & $F$ & ميانكين مجذورات & درجه آزادى & مجموع مجذورات & متغير & واريانس \\
\hline $.1 . .1$ &.$/ F A$ & TEN & 1.1 .4 & 1 & $1.1 / . r$ & اضطراب امتحان & \multirow{4}{*}{ كروه } \\
\hline.$/ \ldots 1$ & . & $1 W / \Delta$ & $11 D / \pi$ & 1 & $11 D / \pi$ & خودكارآمدى & \\
\hline $.1 . .1$ &.$/ p r$ & TITI & $\Delta F / T A$ & 1 & $\Delta F / T A$ & كمال كرايع & \\
\hline \multirow[t]{5}{*}{$\%$} &.$/ M A$ & $11 / r$ & $r / P$ & 1 & $r r / P$ & عزت نفس & \\
\hline & & & $r / \mathrm{rA}$ & rA & $1.0 / 9 r$ & اضطراب امثحان & \multirow{4}{*}{ خطا } \\
\hline & & & Nar & rA & VINa & خودكارآمدى & \\
\hline & & & r/ar & rA & $V / / Y$ & كمال كرايع & \\
\hline & & & $1 / 4$ & $r \Lambda$ & $\Delta \Delta / F \xi$ & عزت نفس & \\
\hline
\end{tabular}




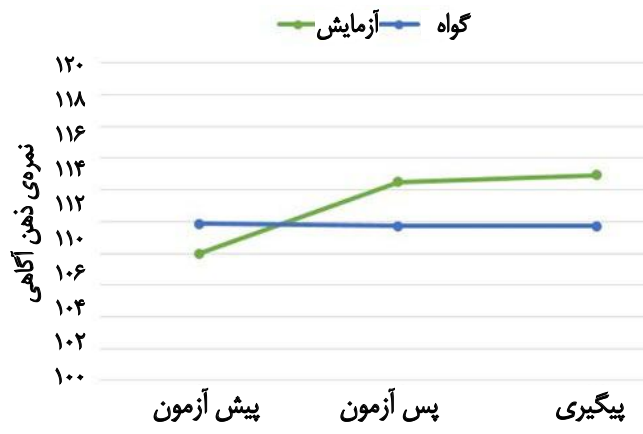

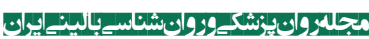

تصوير ا. اثر تعامل كروه و موقعيت در نمره ذهن آكاهى تحصيلى

با توجه به جدول شماره ب نتايج سطح معنادارى نشان ميدهد إندان

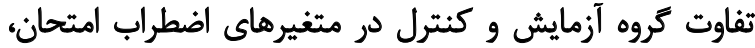

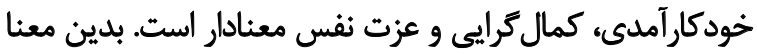

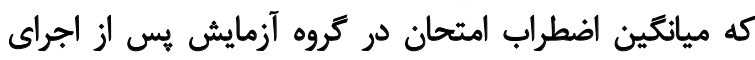

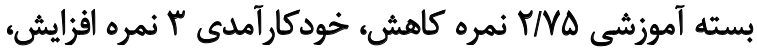

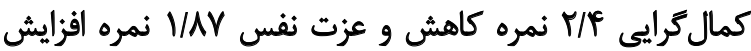

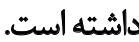

نثايج سطح معنادارى نشان مى دهد ميانكين هاي كروه آزمايش وكنترل در متغيرهاى اضطراب امتحان، خودكارا آمدى، كمال كرايى كروائ

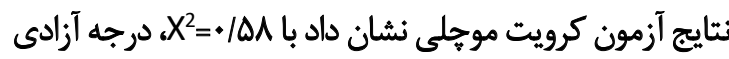

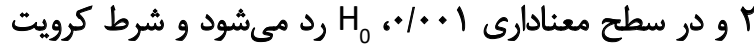

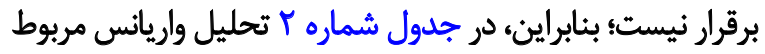

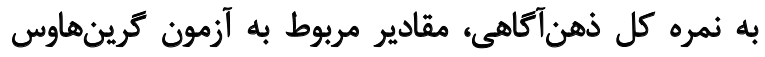
كيسر ها آورده شده است.

نتايج جدول شماره Y نشان مى دهد اثر اصلى موقعيت آزمايش

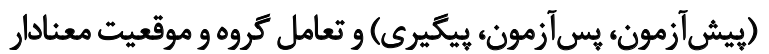

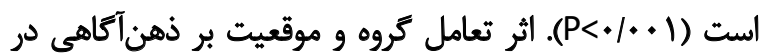

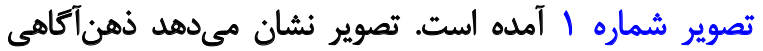

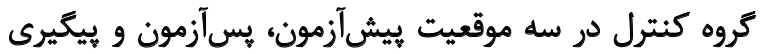

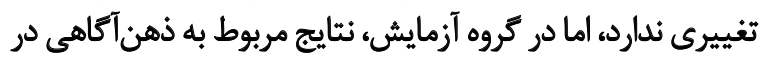
يس آزمون و بيكيرى بيشتر از كروه كنترل است.

حودكار آمدي، عزت نفس، اضطراب امثحان و كمال مَّرايي

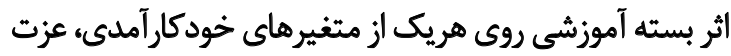

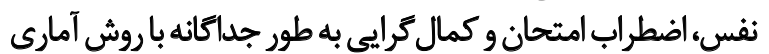

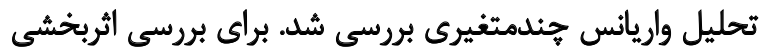

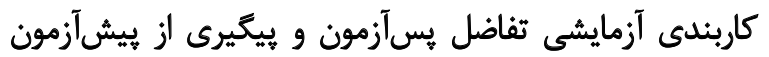

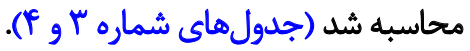

25. Green House-Geisser

جدول F. تحليل واريانس جندمتغيرى تغيير اضطراب امتحان، خودكارآمدى، كمال كرايح و عزت نفس در بيغيرى

\begin{tabular}{|c|c|c|c|c|c|c|c|}
\hline سطح معنادارى & اندازه اثر & $F$ & ميانكين مجذورات & درجه آزادى & مجموع مجذورات & متغير & واريانس \\
\hline $.1 . .1$ &.$p q$ & Tr/QS & $111 / 8$ & 1 & $111 / 8$ & اضطراب امثحان & \\
\hline $.1 . .1$ &.$M$ & NVE & $V \Delta / \cdot 1$ & 1 & $V \Delta / \cdot 1$ & خودكارآهدى & \\
\hline .1 .01 & .180 & $\Delta T / T$ & PAYT/ & 1 & PAT/K & كمال كرايي & كروه \\
\hline \multirow[t]{5}{*}{.1 .08} &.$/ M$ & $11 / \pi$ & rT/RT & 1 & TYRT & عزت نفس & \\
\hline & & & e/rr & rA & $110 / A^{2}$ & اضطراب امتحان & \\
\hline & & & $N \Delta \Delta$ & rA & Hrq/Fa & خودكارآمدى & 46 \\
\hline & & & Q/m & rA & TANEA & كمال كرايى & ح \\
\hline & & & $1 / 4$ & rA & $\Delta \Delta / \digamma$ & عزت نفس & \\
\hline
\end{tabular}

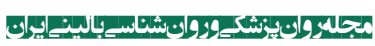

جدول هـ نتايج تحليل واريانس آميخته براى نمره كلى اهمال كارى تحصيلى

\begin{tabular}{|c|c|c|c|c|c|c|}
\hline اندازه اثر & سطح معنادارى & $\mathbf{F}$ & ميانكين مجذورات & درجه أزادى & مجموع مجذورات & منبع واريانس \\
\hline .1810 & $<+/ *+1$ & $\Delta F / \backslash \Delta$ & $|r| F A$ & $1 / 8 \Delta$ & $r+1 / 19$ & موقعيت \\
\hline \multirow[t]{2}{*}{$.18 \Delta 9$} & $<\cdot 1 \cdot+1$ & $N E / g V$ & $\mid+* / M 1$ & 1/8ब & $\mid \$ 91.1$ & موقعيت×كروه \\
\hline & & & $T / M F$ & Pe/mA & $1.7 \%$ & خط \\
\hline
\end{tabular}

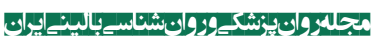


كمال كرايي و درنتيجه كاهش اهمال كارى تحصيلى اثر دارد. اين

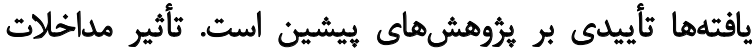

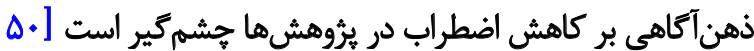

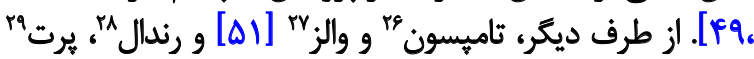

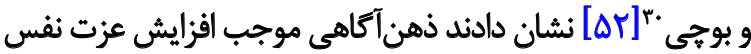

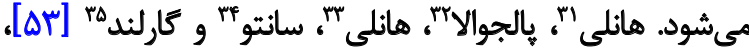

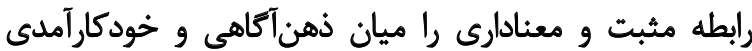

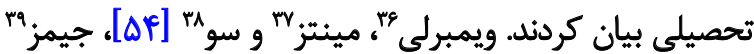

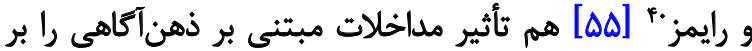

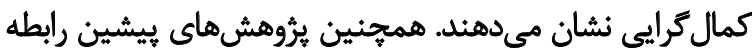

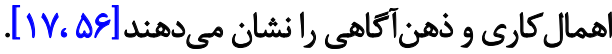

ذهن آكاهى به عنوان حالت توجه برانكيخته و آكاهى از آنجهه

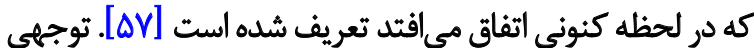

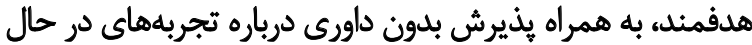

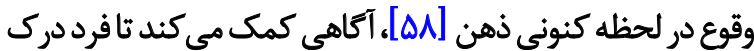

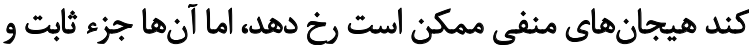

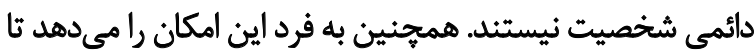

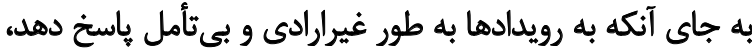

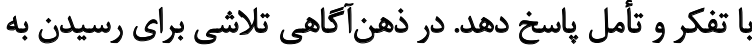

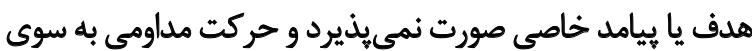

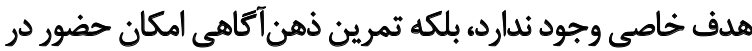

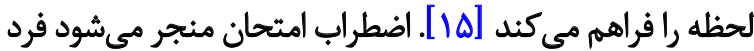

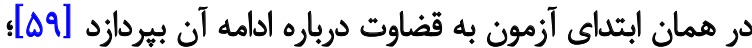

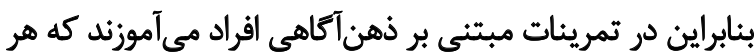

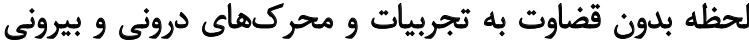

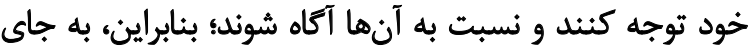

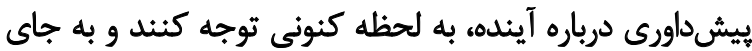

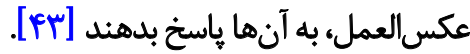
علاوه بر اين، كابات زين بيان مىكند كه مىتوان نكرشها و

26. Thompson

27. Waltz

28. Randall

29. Pratt

30. Bucci

31. Hanley

32. Palejwala

33. Hanley

34. Canto

35. Garland

36. Wimberley

37. Mintz

38. Suh

39. James

40. Rimes

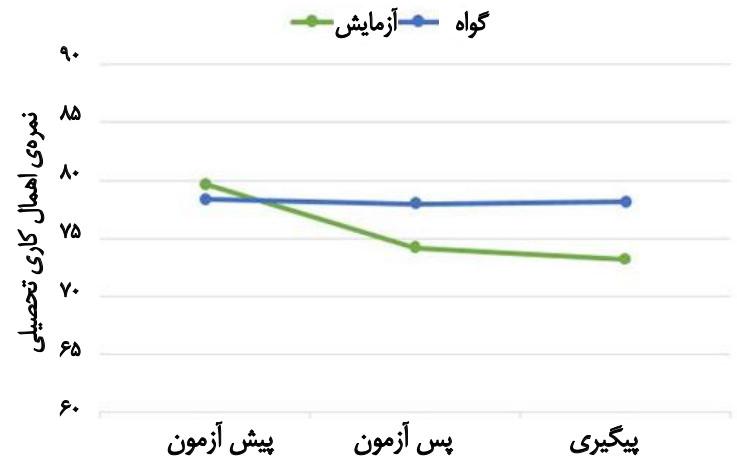

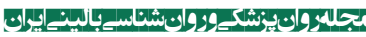

تصوير Y. اثر تعامل گروه و موقعيت در نمره كل اهمال كارى تحصيلى

و عزت نفس در آزمون ييخيرى تفاوت معنادار دارد. بدين معنا كه

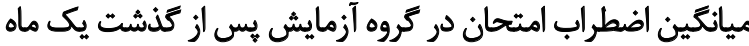

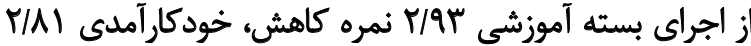

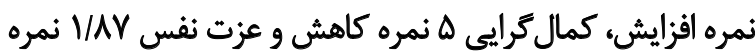
افزايش داشته است. ا الهمال كارى تحصيلى

ابتدا براي بررسى معنادارى اهمال كارى تحصيلى در بيش آزمون

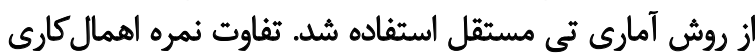

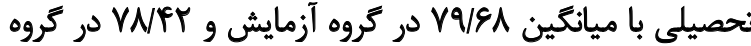

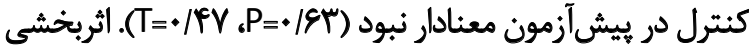

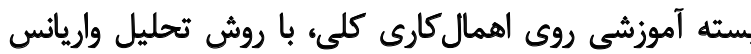
آميخته، بررسى شد. نتايج در جدول شماره ه ه و تصوير شماره

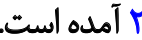

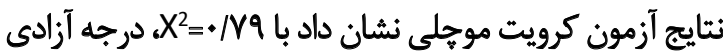

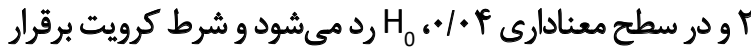

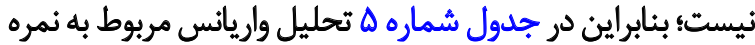

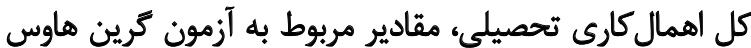

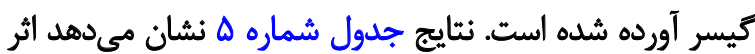

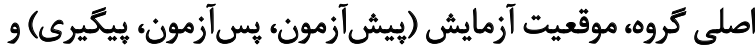

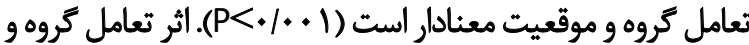
موقعيت بر اهمال كارى در تصوير شماره ب آمده است.

تصوير نشان مى دهد اهمال كارى تحصيلى تروه كنترل در سه

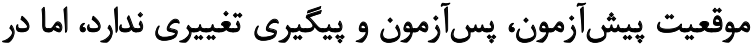

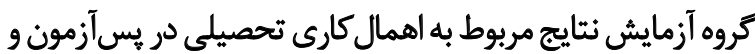
ييكيرى كمتر از كروه كنترل است.

بحث

نتايج يُروهش نشان داد بسته آموزشى مبتنى بر ذهن آكاهي

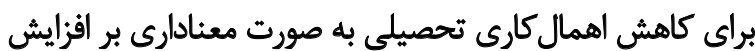

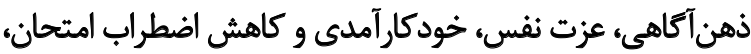


اين تمرينها را خود نيز در خارج از كروه انجام دهد مطابق انتظار

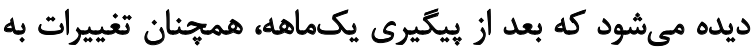
شكل معنادارى يايدار هستند كه نشان از يايدارى اثر آموزش دارئ دارد.

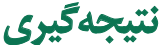

درمجموع، مبانى نظرى و بروهشى مربوط به به اثركذارى

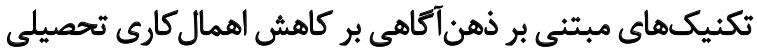

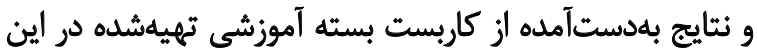

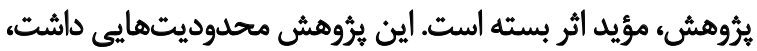

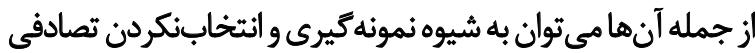

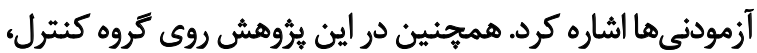

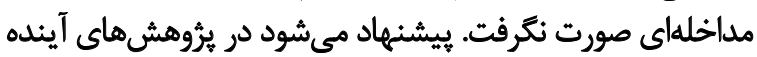

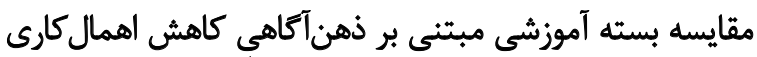

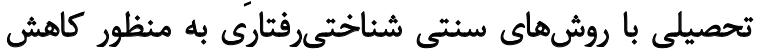

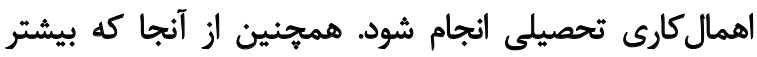

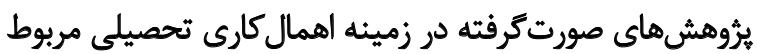

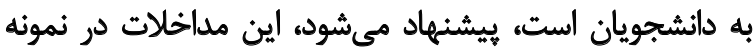

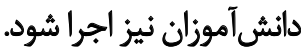

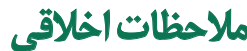

\section{يبروى از اصول اخلاق يثوهش}

همه اصول اخلاقى در اين مقاله رعايت شده است.

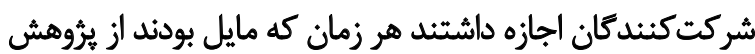

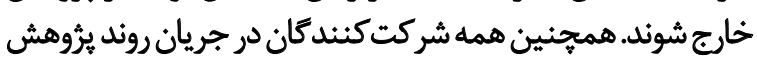
بودند. اطلاعات آنها محرمانه نكَه داشته شد شد.

$$
\text { حامي مالي }
$$

اين مقاله از يايان نامه دكتراى نويسنده اول در كروه روانشناسى،

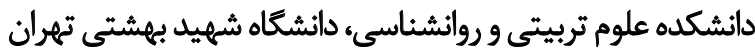
كرفته شده است.

$$
\text { مشاركت نويسند مكان }
$$

مفهومسازى: تمام نويسندكان؛ روش شناسى: محمود حيدرى،

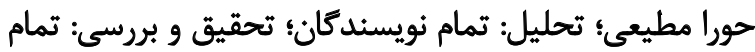

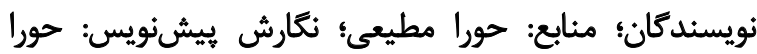

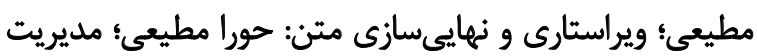

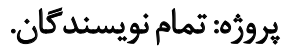

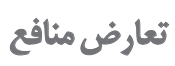

بنابر اظهار نويسندكان، اين مقاله تعارض منافع ندارد.
هيجانات منفى درباره موقعيتهاي مختلف را از طريق مشاهده

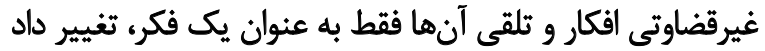

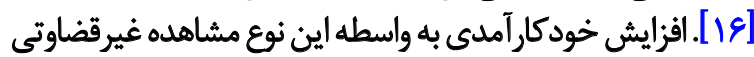

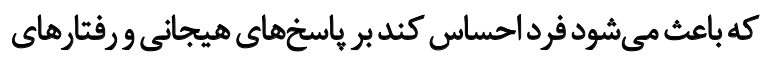

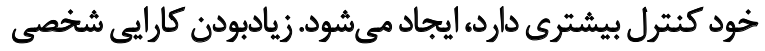

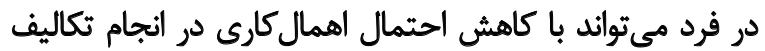

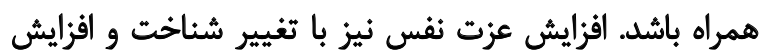

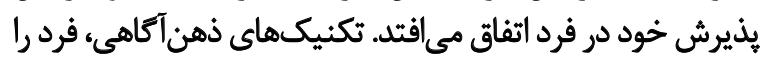

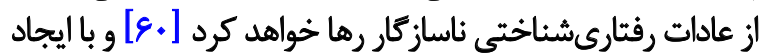

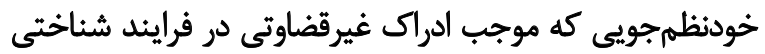

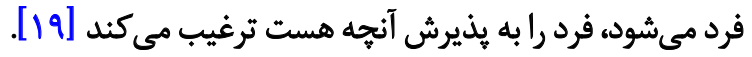

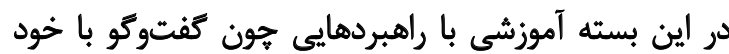

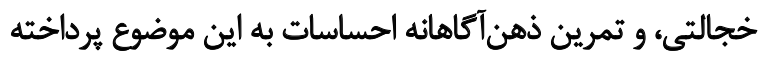

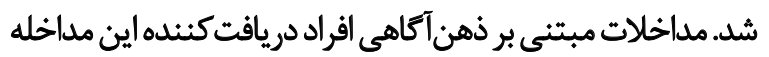

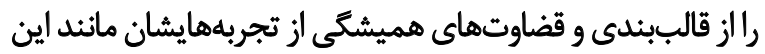

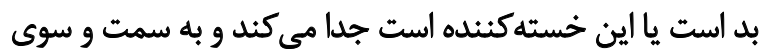

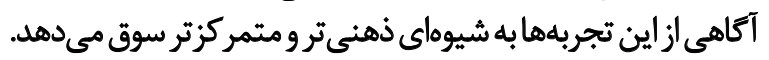
هدف از اين كار اين است كه در ائها

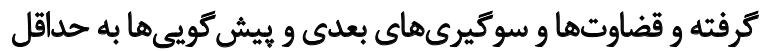

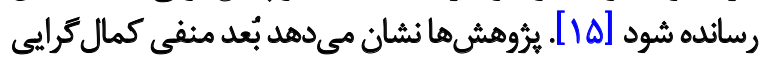

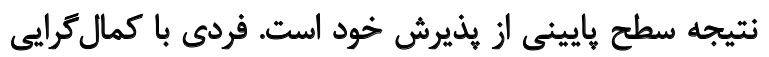

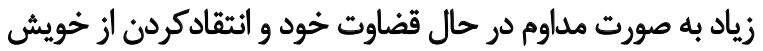

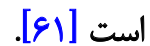

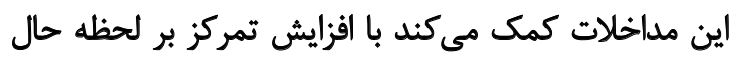

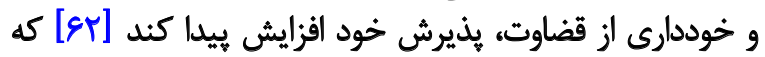

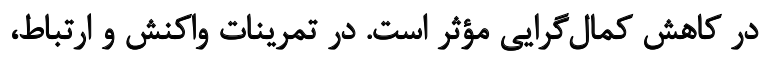

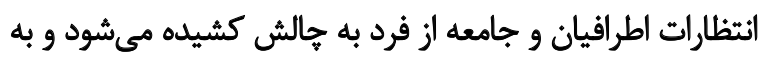

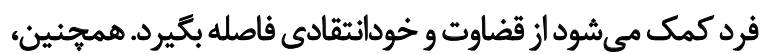

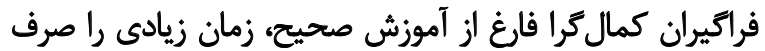

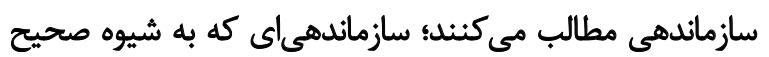

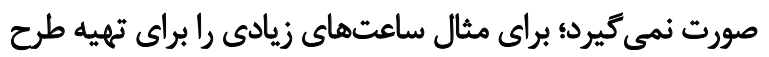

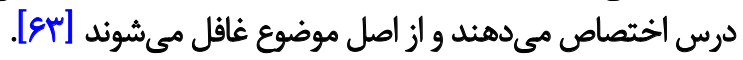
تمرينهاى ذهنئكآهى با بيشترشدن هشيارى و و ثذيرش

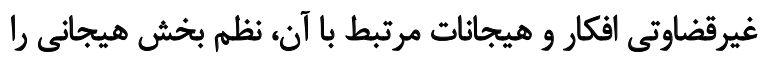

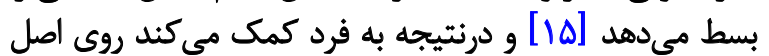

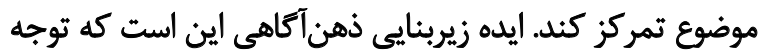

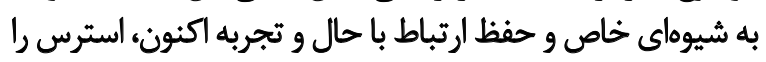

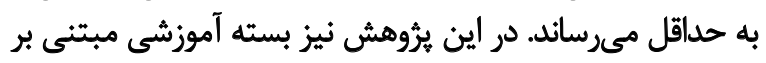

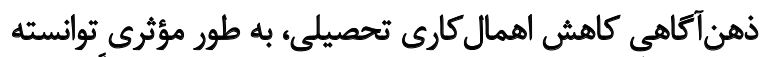

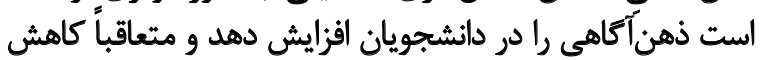

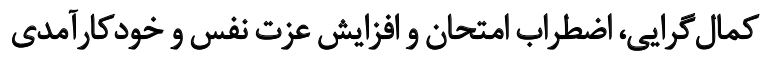

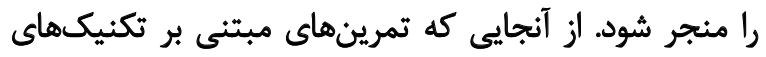

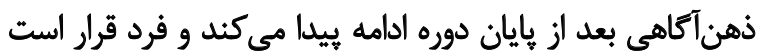




\section{References}

[1] Pychyl TA, Morin RW, Salmon BR. Procrastination and the planning fallacy: An examination of the study habits of university students. Journal of Social Behavior and Personality. 2000; 15(5):135-50

[2] Ferrari JR. Trait procrastination in academic settings: An overview of students who engage in task delays. In Schouwenburg HC, Lay CH, Pychyl TA, Ferrari JR, editors. Counseling the Procrastinator in Academic Settings. Washington: American Psychological Association; 2004

[3] Steel P, Klingsieck KB. Academic procrastination: Psychological antecedents revisited. Australian Psychologist. 2016; 51(1):36-46. [DOI:10.1111/ap.12173]

[4] Kim KR, Seo EH. The relationship between procrastination and academic performance: A meta-analysis. Personality and Individual Differences. 2015; 82:26-33. [DOI:10.1016/j.paid.2015.02.038]

[5] Essau, C, Uzun, B. Procrastination. In Wenzel A, editors. The SAGE encyclopedia of abnormal and clinical psychology. Thousand Oaks: Sage; 2017. [DOI:10.4135/9781483365817]

[6] Ferrari JR, Ferrari JR. Still procrastinating: The no-regrets guide to getting it done. Hoboken: Wiley; 2010.

[7] Ozer BU, Demir A, Ferrari JR. Reducing academic procrastination through a group treatment program: A pilot study. Journal of Rational-Emotive \& Cognitive-Behavior Therapy. 2013; 31(3):12735. [DOI:10.1007/s10942-013-0165-0]

[8] Grunschel C, Patrzek J, Fries S. Exploring reasons and consequences of academic procrastination: An interview study. European Journal of Psychology of Education. 2013; 28(3):841-61. [DOI:10.1007/s10212-012-0143-4]

[9] Patrzek J, Grunschel C, Fries S. Academic procrastination: The perspective of university counsellors. International Journal for the Advancement of Counselling. 2012; 34(3):185-201. [DOI:10.1007/ s10447-012-9150-z]

[10] Uzun B, Ferrari JR, LeBlanc S. Put aside procrastination: Positive emotional outcomes from self-forgiveness for delays. North American Journal of Psychology. 2018; 20(1):171-86

[11] Ellis, A, knaus WJ. Overcoming procrastination. Sydney: Signet, 2002.

[12] Dionne F, Gagnon J, Carbonneau N, Hallis L, Grégoire S, Balbinotti $\mathrm{M}$. Using acceptance and mindfulness to reduce procrastination among university students: Results from a pilot study. Revista Prâksis. 2016; 13(1):8-20. [DOI:10.25112/rp.v1i0.431]

[13] Hayes SC, Villatte M, Levin M, Hildebrandt M. Open, aware, and active: Contextual approaches as an emerging trend in the behavioral and cognitive therapies. Annual Review of Clinical Psychology. 2011; 7:141-68. [DOI:10.1146/annurevclinpsy-032210-104449] [PMID]

[14] Hayes SC, Strosahl KD, Wilson KG. Acceptance and commitment therapy: The process and practice of mindful change. New York City: Guilford Press; 2011.

[15] Saltzman A, Goldin P. Mindfulness-based stress reduction for school-age children. In Greco LA, Hayes SC, editors. Acceptance and Mindfulness Treatments for Children and Adolescents: A Practitioner's Guide. Oakland: New Harbinger; 2008.
[16] Kabat-Zinn J. Full catastrophy living. New York: Delta Trade; 1990.

[17] Sirois FM, Tosti N. Lost in the moment? An investigation of procrastination, mindfulness, and well-being. Journal of Rational-Emotive \& Cognitive-Behavior Therapy. 2012; 30(4):237-48. [DOI:10.1007/s10942-012-0151-y]

[18] Flett GL, Blankstein KR, Martin TR. Procrastination, negative self-evaluation, and stress in depression and anxiety. In Ferrar JR, Johnson JL, McCown WG. Procrastination and Task Avoidance. New York: Plenum Press; 1995. [DOI:10.1007/978-1-48990227-6_7]

[19] Teasdale JD, Segal Z, Williams JM. How does cognitive therapy prevent depressive relapse and why should attentional control (mindfulness) training help. Behaviour Research and Therapy. 1995; 33(1):25-39. [DOI:10.1016/0005-7967(94)E0011-7]

[20] Sirois FM. Procrastination and intentions to perform health behaviors: The role of self-efficacy and the consideration of future consequences. Personality and Individual Differences. 2004; 37(1):115-28. [DOI:10.1016/j.paid.2003.08.005]

[21] Brown KW, Ryan RM, Creswell JD. Mindfulness: Theoretical foundations and evidence for its salutary effects. Psychological Inquiry. 2007; 18(4):211-37. [DOI:10.1080/10478400701598298]

[22] Cutshall JL. Procrastinating in college: Students' readiness and resistance to change. Journal of College Student Development. 2018; 59(4):498-504. [DOI:10.1353/csd.2018.0047]

[23] Klassen RM, Ang RP, Chong WH, Krawchuk LL, Huan VS, Wong IY, et al. A cross-cultural study of adolescent procrastination. Journal of Research on Adolescence. 2009; 19(4):799-811. [DOI:10.1111/j.1532-7795.2009.00620.x]

[24] Schraw G, Wadkins T, Olafson L. Doing the things we do: A grounded theory of academic procrastination. Journal of Educational Psychology. 2007; 99(1):12-25. [DOI:10.1037/00220663.99.1.12]

[25] Yang Z, Asbury K, Griffiths MD. An exploration of problematic smartphone use among Chinese university students: Associations with academic anxiety, academic procrastination, self-regulation and subjective wellbeing. International Journal of Mental Health and Addiction. 2018:1-19. [DOI:10.1007/s11469-018-9961-1]

[26] Firoozabad H, Hosseinzadeh Y, Bassaknejad S, Davoodi, I. [Prediction of subscale test anxiety considering behavioral procrastination, decisional procrastination and cognitive avoidance in university students (Persian)]. Iranian Journal of Psychiatry \& Clinical Psychology. 2018; 23(4):424-37. [DOI:10.29252/nirp. ijpcp.23.4.424]

[27] Klassen RM, Krawchuk LL, Rajani S. Academic procrastination of undergraduates: Low self-efficacy to self-regulate predicts higher levels of procrastination. Contemporary Educational Psychology. 2008; 33(4):915-31. [DOI:10.1016/j.cedpsych.2007.07.001]

[28] Hajloo N. Relationships between self-efficacy, self-esteem and procrastination in undergraduate psychology students. Iranian Journal of Psychiatry and Behavioral Sciences. 2014; 8(3):42-9. [PMID] [PMCID]

[29] Steel P. The nature of procrastination: A meta-analytic and theoretical review of quintessential self-regulatory failure. Psychological Bulletin. 2007; 133(1):65-94. [DOI:10.1037/0033-2909.133.1.65 [PMID] 
[30] Katz I, Eilot K, Nevo N. "I'll do it later": Type of motivation, self-efficacy and homework procrastination. Motivation and Emotion. 2014; 38(1):111-9. [DOI:10.1007/s11031-013-9366-1]

[31] Motie H, Heidari M, Sadeghi MA. Predicting academic procrastination during self-regulated learning in Iranian first grade high school students. Procedia-Social and Behavioral Sciences. 2012; 69:2299-308. [DOI:10.1016/j.sbspro.2013.02.023]

[32] Çapan BE. Relationship among perfectionism, academic procrastination and life satisfaction of university students. ProcediaSocial and Behavioral Sciences. 2010; 5:1665-71. [DOI:10.1016/j. sbspro.2010.07.342]

[33] Pychyl TA, Flett GL. Procrastination and self-regulatory failure: An introduction to the special issue. Journal of Rational-Emotive \& Cognitive-Behavior Therapy. 2012;30(4):203-12. [DOI: 10.1007/ s10942-012-0149-5]

[34] Solomon LJ, Rothblum ED. Academic procrastination: Frequency and cognitive-behavioral correlates. Journal of Counseling Psychology. 1984; 31(4):503-9. [DOI:10.1037/0022-0167.31.4.503]

[35] Hossein Chari M, Dehghani Y. [Prediction of academic failure based on self-regulatory strategies in learning (Persian)]. Journal of Research in Educational Systems. 2008; 2(4):63-74.

[36] Yazdani F. [Test anxiety and academic performance in female nursing students (Persain)]. Quarterly Journal of Nursing Management. 2012; 1(1): 47-58.

[37] Besharat MA, Azizi K, Poursharifi H. The relationship between parenting styles and children's perfectionism in a sample of Iranian families. Procedia-Social and Behavioral Sciences. 2011; 15:1276-9. [DOI:10.1016/j.sbspro.2011.03.276]

[38] Sherer M, Maddux JE, Mercandante B, Prentice-Dunn S, Jacobs B, Rogers R. The self-efficacy scale: Construction and validation. Psychological Reports. 1982; 51(2):663-71. [DOI:10.2466/ pr0.1982.51.2.663]

[39] Najafi M, Foladjang M. [The relationship between self-efficacy and mental health among high school students (Persian)]. Journal of Clinical Psychology \& Personality. 2007; 1(22):69-83.

[40] Mansouri Sepehr R, Khodapanahi Mk, Heidari M. [Cognitive dissonance: The effect of self-centered self-esteem moderation on changing attitudes (Persian)]. Journal of Applied Psychology. 2012; 6(2):25-40.

[41] Baer RA, Smith GT, Hopkins J, Krietemeyer J, Toney L. Using self-report assessment methods to explore facets of mindfulness. Assessment. 2006; 13(1):27-45. [DOI:10.1177/1073191105283504] [PMID]

[42] Neuser NJ. Examining the factors of mindfulness: A confirmatory factor analysis of the five facet mindfulness questionnaire. [PhD. thesis]. Forest Grove, Oregon: Pacific University; 2010.

[43] Ahmadvand Z, Heydarinasab L, Shairi MR. [An investigation of the validity and reliability of psychometric characteristics of five facet mindfulness questionnaire in Iranian non-clinical samples (Persian)]. Journal of Behavioral Sciences. 2013; 7(3):229-37.

[44] Kabat-Zinn J. Mindfulness-based stress reduction. Constructivism in the Human Sciences. 2003; 8(2):73-107.

[45] Saltzman A, Santorelli S. A still quiet place: A mindfulness program for teaching children and adolescents to ease stress and difficult emotions. Oakland, California: New Harbinger; 2014.
[46] Siegel RD. The mindfulness solution: Everyday practices for everyday problems. New York City: Guilford Press; 2009.

[47] Gyoerkoe K, Wiegartz P. Theworrier's guide to overcoming procrastination: Breaking free from the anxiety that holds you back. Oakland, California: New Harbinger; 2011.

[48] Cullen M, Pons GB. The mindfulness-based emotional balance workbook: An eight-week program for improved emotion regulation and resilience. Oakland, California: New Harbinger; 2015.

[49] Beauchemin J, Hutchins TL, Patterson F. Mindfulness meditation may lessen anxiety, promote social skills, and improve academic performance among adolescents with learning disabilities. Complementary Health Practice Review. 2008; 13(1):34-45. [DOI:10.1177/1533210107311624]

[50] Woodruff SC, Glass CR, Arnkoff DB, Crowley KJ, Hindman RK, Hirschhorn EW. Comparing self-compassion, mindfulness, and psychological inflexibility as predictors of psychological health. Mindfulness. 2014; 5(4):410-21. [DOI:10.1007/s12671-0130195-9]

[51] Thompson BL, Waltz J. Everyday mindfulness and mindfulness meditation: Overlapping constructs or not? Personality and Individual Differences. 2007; 43(7):1875-85. [DOI:10.1016/j. paid.2007.06.017]

[52] Randal C, Pratt D, Bucci S. Mindfulness and self-esteem: A systematic review. Mindfulness. 2015; 6(6):1366-78. [DOI:10.1007/ s12671-015-0407-6]

[53] Hanley AW, Palejwala MH, Hanley RT, Canto AI, Garland EL. A failure in mind: Dispositional mindfulness and positive reappraisal as predictors of academic self-efficacy following failure. Personality and Individual Differences. 2015; 86:332-7. [DOI:10.1016/j.paid.2015.06.033]

[54] Wimberley TE, Mintz LB, Suh H. Perfectionism and mindfulness: effectiveness of a bibliotherapy intervention. Mindfulness. 2016; 7(2):433-44. [DOI:10.1007/s12671-015-0460-1]

[55] James K, Rimes KA. Mindfulness-based cognitive therapy versus pure cognitive behavioural self-help for perfectionism: A pilot randomised study. Mindfulness. 2018; 9(3):801-14. [DOI:10.1007/ s12671-017-0817-8] [PMID] [PMCID]

[56] Kervin CE, Barrett HE. Emotional management over time management: Using mindfulness to address student procrastination. Journal of Writing Center Scholarship. 2018; 42(9-10):10-8.

[57] Walsh JJ, Balint MG, SJ DR, Fredericksen LK, Madsen S. Predicting individual differences in mindfulness: The role of trait anxiety, attachment anxiety and attentional control. Personality and Individual differences. 2009; 46(2):94-9. [DOI:10.1016/j. paid.2008.09.008]

[58] Leigh J, Bowen S, Marlatt GA. Spirituality, mindfulness and substance abuse. Addictive Behaviors. 2005; 30(7):1335-41. [DOI:10.1016/j.addbeh.2005.01.010] [PMID]

[59] Schutz PA, Davis HA. Emotions and self-regulation during test taking. Educational Psychologist. 2000; 35(4):243-56. [DOI:10.1207/S15326985EP3504_03]

[60] Brown KW, Ryan RM. The benefits of being present: Mindfulness and its role in psychological well-being. Journal of Personality and Social Psychology. 2003; 84(4):822-48. [DOI:10.1037/00223514.84.4.822] [PMID] 
[61] Short MM, Mazmanian D. Perfectionism and negative repetitive thoughts: Examining a multiple mediator model in relation to mindfulness. Personality and Individual Differences. 2013. 55(6):716-21. [DOI:10.1016/j.paid.2013.05.026]

[62] Fletcher L, Hayes SC. Relational frame theory, acceptance and commitment therapy, and a functional analytic definition of mindfulness. Journal of Rational-Emotive and Cognitive-Behavior Therapy. 2005; 23(4):315-36. [DOI:10.1007/s10942-005-0017-7]

[63] Motie H, Heidari M, Sadeghi MA. Development of a self-regulation package for academic procrastination and evaluation of its effectiveness. The European Journal of Social \& Behavioural Sciences. 2013; 4(1):889-96. 
This Page Intentionally Left Blank 